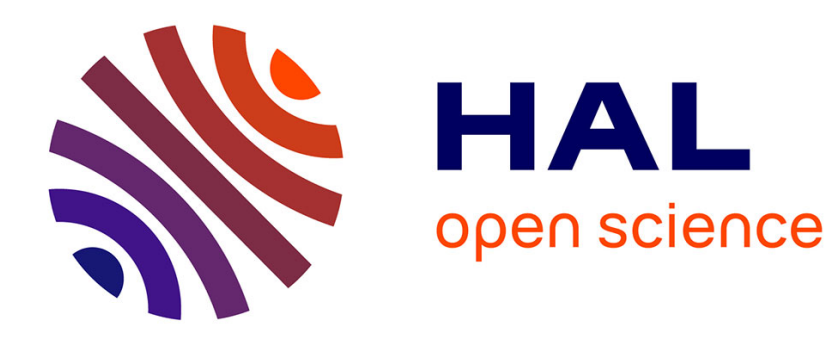

\title{
Extremal Hamiltonian for Directed Polymers
}

Stéphane G. Roux, Yi-Cheng Zhang

\section{To cite this version:}

Stéphane G. Roux, Yi-Cheng Zhang. Extremal Hamiltonian for Directed Polymers. Journal de Physique I, 1996, 6 (3), pp.385-392. 10.1051/jp1:1996163 . jpa-00247191

\section{HAL Id: jpa-00247191 https://hal.science/jpa-00247191}

Submitted on 1 Jan 1996

HAL is a multi-disciplinary open access archive for the deposit and dissemination of scientific research documents, whether they are published or not. The documents may come from teaching and research institutions in France or abroad, or from public or private research centers.
L'archive ouverte pluridisciplinaire HAL, est destinée au dépôt et à la diffusion de documents scientifiques de niveau recherche, publiés ou non, émanant des établissements d'enseignement et de recherche français ou étrangers, des laboratoires publics ou privés. 


\title{
Extremal Hamiltonian for Directed Polymers
}

Stéphane Roux $\left({ }^{1, *}\right)$ and Yi-Cheng Zhang $\left({ }^{2}\right)$

$\left({ }^{1}\right)$ Laboratoire de Physique et Mécanique des Milieux Hétérogènes, École Supérieure de Physique et Chimie Industrielles, 10 rue Vauquelin, 75231 Paris Cedex 05, France

$\left({ }^{2}\right)$ Institut de Physique Théorıque, Université de Fribourg, Pérolles, CH-1700, Fribourg, Switzerland

(Recerved 7 September 1995, recelved in final form 15 November 1995, accepted 27 November 1995)

PACS.61.41.te - Polymers, elastomers, and plastics

PACS.05.40. $+\mathrm{j}$ - Fluctuation phenomena, random processes, and Brownian motion

PACS.64.60.Ak - Renormalization-group, fractal, and percolation studies of phase transitions

\begin{abstract}
We study an extremal Hamiltonian for directed polymers. The ground states are degenerate and they form a cluster of directed percolation. This degeneracy has a hierarchical fractal structure and can be lifted by a progressive optimization.
\end{abstract}

In statistical mechanics, there are recently two topics which attracted much attention: one is directed polymer in random media [1] and the other is older, directed percolation [2]. The two models are defined apparently very differently and for completely different purposes. Only very recently their deep relationship has been revealed and in this note we shall further explore this subtle relation.

Recent progress in connection with other dynamical interface problems reveals however that the directed percolation clusters arise inconspicuously but surprisingly for the Self-Organised Depinning (SOD) [3-5] interface model as well as for the KPZ equation [6] in quenched disorder. [7-9] It is found that when the interface's updating rule is extremal, i.e. only the largest or smallest value is elected, directed percolation clusters appear naturally and the scaling exponents can be understood by a systematic scaling theory $[10,11]$. In the Bak-Sneppen evolution model [12], directed percolation again appear in a hidden way [13]. Extremal updating rules usually correspond to the zero temperature limit of the equilibrium physics. While the above models are from dynamics, we address in this Letter extremal physics in equilibrium statistics where directed percolation appears naturally.

The directed polymer problem is defined through the Hamltonian in a $d+1$ lattice

$$
\mathcal{H}_{1}(\mathcal{P})=\sum_{\mathbf{x} \in \mathcal{P}} \eta(\mathbf{x})
$$

where $\mathcal{P}$ is a directed path, and $\eta(\mathbf{x})$ is a random value assigned to the site $\mathbf{x}$ and is independently distributed through the lattice. For our purpose $\eta$ takes values uniformly in $[0,1]$. The

$\left(^{*}\right)$ Author for correspondence (e-mall: roux@pmmh.espc1.fr) 
partition function is

$$
\mathcal{Z}=\sum_{\mathcal{P}} \exp (-\mathcal{H}(\mathcal{P}) / T)
$$

where the sum is over all the directed paths with the same end points. At the zero temperature limit, only one path is selected which is called the ground state or the optimal path. The transverse deviation and energy fluctuation exponents are known exactly in $2 \mathrm{~d}(2 / 3,1 / 3)$ and they remain the same at finite temperatures.

Let us define now an extremal version of the directed polymer Hamiltonian (1). Instead of contributions from every site on the path, we let only the largest value represent the entire path:

$$
\mathcal{H}_{m}(\mathcal{P})=\max _{\mathbf{x} \in \mathcal{P}} \eta(\mathbf{x})
$$

This Hamiltonian is an intensive variable compared to the traditional extensive variable. Therefore no traditional thermodynamics will arise. We are interested here only in the zero temperature limit. At $T=0$ our Hamltonian serves a bookkeeping device to classify the degenerate ground states which can extended to other extremal models as well. In the extremal Hamiltomian (3) any given configuration has a well defined energy. The only novelty here is that the ground state degeneracies are inevitable and below we shall show these degeneracies follow a hierarchical fractal structure.

The physical motivation for the extremal Hamiltonıan can be illustrated by an example: consider a path going through a random landscape. A traditional optimal directed polymer will minimize the total cost - i.e. the sum of the random variables (1). We can consider the problem of finding the path where the highest (or lowest) point is all we want to minimize - this is described by equation (3). For instance, for the water level in a random landscape only the highest pass matters. Such examples can also be found in electron transport where bottleneck potential decides whether a state is conducting or not [14].

The above considerations were at the basis of the interesting work by Cieplack, Maritan and Banavar [15]. They proposed a simular extremal model for the case of a non directed path, in which backward steps are allowed. They showed that the optimal path had a fractal structure whose dimension was studied numerically and the ultrametric tree structure was explained.

In the zero temperature limit, the energy of directed paths using Hamitonian (3) converges toward the (site) directed percolation threshold $p_{\mathrm{c}}$. To show this property, simply imagine removing sites with $\eta$ larger than a threshold $p$. If $p<p_{c}$ there is no continuous directed path across the lattice, hence $\mathcal{H}_{m} \geq p_{\mathrm{c}}$. At and above threshold $p \geq p_{\mathrm{c}}$ there is a directed path spanning through the lattice. At zero temperature, the minimum energy is selected so that 1t coincides exactly with the percolation threshold. From this observation, we can deduce the exponent governing the scaling of relative energy fluctuations with the system size as identical to the percolation threshold fluctuations in a finite size system (1.e. $1 / \nu_{\|} \approx 0.577-$ for a lattice with a fixed aspect ratio - as compared to the usual $2 / 3$ value for the DP case).

Mathematically, we can define a Hamlltonian interpolating (1) and (3)

$$
\mathcal{H}_{\alpha}(\mathcal{P})=\sum_{\mathrm{x} \in \mathcal{P}} \eta^{1 / \alpha}(\mathrm{x})
$$

Even though this is still a sum (extensive variable), in the $\alpha \rightarrow 0^{+}$limit the highest value plays a dominant role. More precisely $\left(\mathcal{H}_{\alpha}\right)^{\alpha} \rightarrow \mathcal{H}_{m}$. For a systematical study see reference [16]. Note this Hamiltonian allows to consider the usual thermodynamics, and this is an obliged step for extracting the proper transformation to apply to any thermodynamic variable in order to extract the relevant non degenerate limit when $\alpha$ approaches 0 . However, as previously 


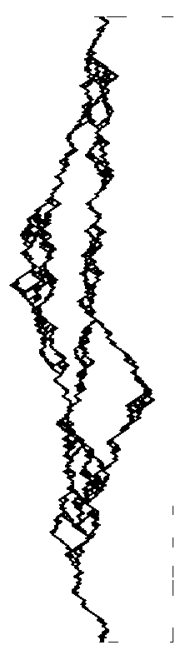

a

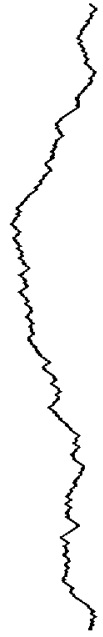

b

Fig. 1. - a) Set of paths which have the same energy using expression (4). The high degeneracy of this ground state appears in the form of fractal "blobs". The lattice size is 800 along the paths and 200 in a perpendicular direction. b) The optimal path for the same disorder as in the previous Figure using the reduction of degeneracy procedure.

mentioned we essentially focus on the zero temperature limit in this letter, and thus we can deal directly with the form (3).

The ground states of an extremal Hamiltonian are necessarily degenerate since there are different paths sharıng the same highest site. In fact the ground states are so degenerate that the ensemble of the optimal paths forms no longer a one-dimensional object but rather a directed percolation cluster with dangling ends removed, hereafter referred to as a directed "backbone". Figure 1a illustrates such a set of paths.

The degeneracy of the optimal paths is not really so if we consider further optimizations. Two paths sharing the same highest point may have different second highest points. If we require that the second highest point has to be the smallest among all the degenerate paths, the degeneracy is reduced. This requirement is natural if we consider the Hamiltonian (4) for infinitesimal $\alpha$ and compare the energy of two paths in the ground state of Hamiltoman (3), before taking the limit $\alpha \rightarrow 0^{+}$In fact we may apply this optimization recursively down to the last site of the path. Thus we can completely eliminate the degeneracy and find a unique Id path which survives this hierarchical optimization procedure. Figure $1 \mathrm{~b}$ shows the resulting optimal path after the degeneracy reduction starting from the geometry of Figure 1a.

Curiously in the SOD model [3-5], the interface lies also on a directed percolation cluster, but statistically only a special $1 \mathrm{~d}$ subset of the cluster. This subset corresponds to the opposite limit of the above hierarchical optimization. The interface selects the directed paths which have the highest secondary sites among the degenerate paths. This is due to the particular choices of the extremal updating rules of their model. In fact the second highest secondary sites play a crucial role in the so-called "associated activation process" $[10,11]$ and their statistics has been analyzed in details.

In the limit case $\alpha \rightarrow 0^{+}$, the non-degenerate optimal path lies within a directed percolation cluster, and thus its roughness exponent can be deduced from the correlation length exponents 

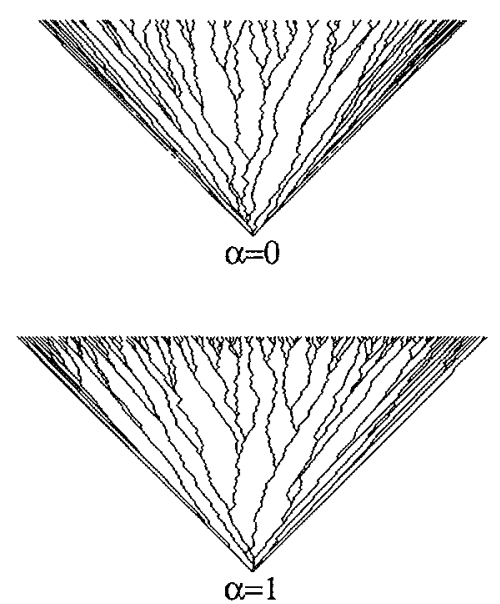

Fig. 2. - Set of optimal paths (using the degeneracy reduction algorithm) which connects the a fixed point to uniformly distributed end points along a line perpendicular to the preferred direction. The length of the paths is 500 .

of the directed percolation problem. More precisely, $\zeta$ has to be smaller or equal to the ratio $\nu_{\perp} / \nu_{\|}$, and thus $\zeta \approx 0.633$ in two dimensions using the known [17] values $\nu_{\|}=1.733$ and $\nu_{\perp}=1.097$. In fact this upper bound is also expected to be a lower bound since the optimization process imposes the large scale roughness in the first steps. It is interesting to note the very small change in the numerical value of the Hurst exponent, $\zeta$, from the standard directed polymer problem and the extremal one.

For our extremal model, we plot the "river network" structure [1] in Figure 2 by computing the set of all optimum paths which connect one fixed point to a series of regularly spaced points on a line perpendicular to the preferred direction of the paths. We observe a similar tree-like structure, with a slight tendency to have more parallel paths than for the DP case [1]. This computation is only presented here for illustration.

The algorithm we used is based on the above construction. We first construct a regular lattice, on the sites or bonds of which we distribute random numbers $x$ unformly distributed between 0 and 1 with no spatial correlations. In the case of bi-periodic boundary conditions we have to first extract the directed backbone at the effective directed percolation threshold of the lattice which can conveniently be done by a transfer matrix algorithm. Then the bond or site which imposes the threshold is identified and its random number is reset to 0 so that it is "forced" in the following optimized structure. Then the new threshold is found and again set to zero up to the stage where the backbone is reduced to a single walk. To accelerate the algorithm it is convenient to identify at all time steps the "blob" structure of the backbone. In this case, the recursive optimization can be done considering each blob independently.

A much more efficient algorithm can be used in the case of non-biperıodic boundary conditions such as is the case for the "ruver network" structure. In the latter case, one simply uses a standard directed invasion percolation algorithm. The latter naturally gives rise to a tree like structure by drawing a link between the "parent site" and its descendent. One can show that the optimal path is simply the first percolating branch which is trivially extracted from the tree structure. The latter algorithm is not restricted to the directed case, and it can be very efficiently extended to the non-directed case. One thus extract the optimal path is a single sweep through the lattice. Non-directed optimal path could be generated on a $1000 \times 1000$ 


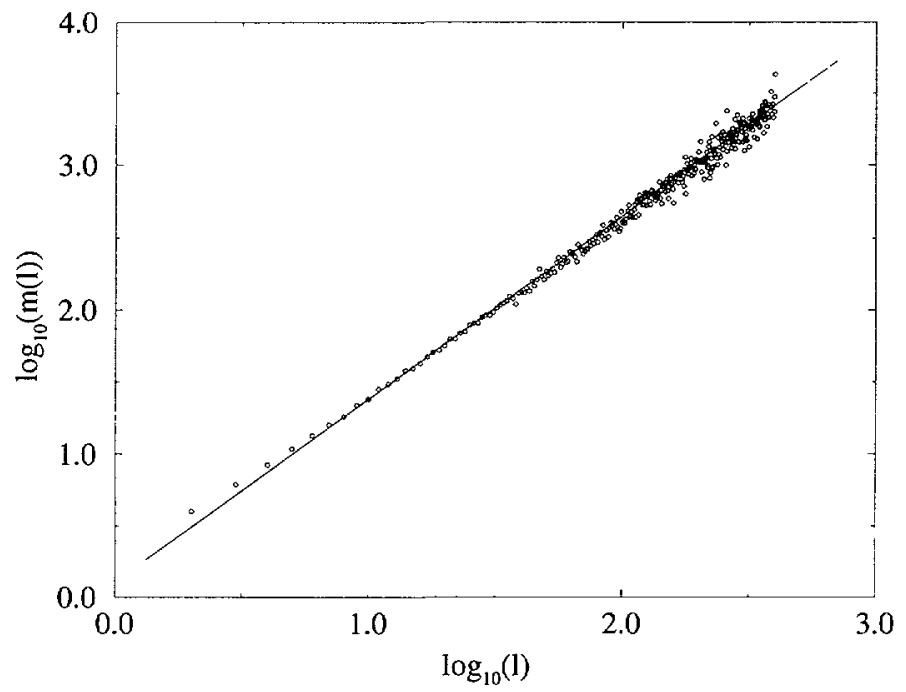

Fig. 3. - Log-log plot of mass versus extension of "blobs of degeneracy". The straight line has a slope 1.27 which gives the fractal dimensions of the blobs.

lattice in about 2 min CPU time on a medium size workstation. The directed case is evidently much faster.

The directed backbone at the first stage can be decomposed in blobs connected to each other by "bottlenecks". Each blob can be characterized by its "mass" $m$ (i.e. the number of sites it contains) and its length $\ell$ along the $y$ axis as computed in the geometry of Figure 1 . Figure 3 shows a log-log plot of the average mass of those blobs as a function of their length. A power-law fit gives an estimate of the fractal dimension $D$ defined as $m \propto \ell^{D}$, with $D \approx 1.27$. These data have been obtained after averaging over 100 lattices of size $800 \times 200$.

The fractal dimension of the directed backbone has been related to the usual critical exponents of directed percolation by Arora et al. [18]. However, a different value is measured here. The reason for this discrepancy comes from the boundary conditions. In the computation of reference [18], the backbone was defined as the intersection of the forward and backward directed clusters starting form two opposite borders of a lattice. In our case, the starting point and end point are single sites, "bottlenecks". This difference can be taken into account in the derivation of the fractal dimension. Let us consider the initial point of a blob as being the origin $(0,0)$ and the end point at coordinates $(0, \ell)$. The probability that a point $(x, y)$ belongs to the forward cluster starting from the origin is proportional to $p_{\mathrm{f}}(x, y) \propto y^{-\beta / \nu_{\|}}$ provided $|x|<y^{\nu_{\perp} / \nu_{\|}}$where $\beta$ is the order parameter critical exponent (in two dimensions, $\beta=0.277$ ). Reversing the preferred direction, we can also obtain the probability that the same site belongs to the backward cluster starting at the end point, $p_{\mathrm{b}}(x, y) \propto(\ell-y)^{-\beta / \nu_{\|}}$provided $|x|<(\ell-y)^{\nu_{\perp} / \nu_{\|}}$

These two probabilities are independent from each other since the two clusters from the orign and from the end point do not overlap, thus the probability that a site $(x, y)$ is part of the backbone can be written $p_{\mathrm{f}}(x, y) p_{\mathrm{b}}(x, y)$. Using the fourfold symmetry of the problem, the mass of the blob can be written as 


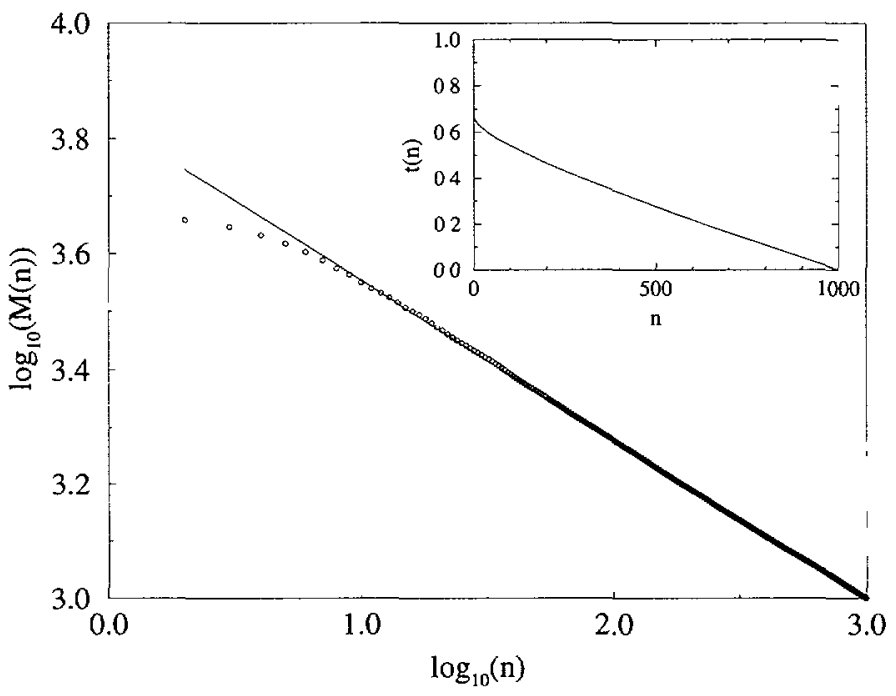

Fig. 4. - Total number of sites which belong to the set of optimal paths as a function of the iteration index for the degeneracy reduction, in a log-log scale. The straight line has a slope -0.28 . The insert shows the threshold of the critical site versus iteration index.

$$
\begin{aligned}
m & \propto \int_{y=0}^{y=\ell / 2} \int_{x=0}^{x=y^{\nu \perp} / \nu_{\|}} y^{-\beta / \nu_{\|}}(\ell-y)^{-\beta / \nu_{\|}} \mathrm{d} x \mathrm{~d} y \\
& \propto \ell^{1+\nu_{\perp} / \nu_{\|}-2 \beta / \nu_{\|}}
\end{aligned}
$$

so that the fractal dimension amounts to

$$
D=\frac{\nu_{\|}+\nu_{\perp}-2 \beta}{\nu_{\|}} \approx 1.31
$$

In good agreement with the measured value 1.27 .

Using this information, we can have access to the progressive reduction of degeneracy in the structure. Let us consider the total number of sites $M(n)$ which belong to the backbone at the $n^{\text {th }}$ iteration. Figure 4 shows a $\log -\log$ plot of $M(n)$ versus $n$. We do observe a power-law behavior for $6<n<1000$ with an exponent measured to be $M(n) \propto n^{-0.28}$. At the $n^{\text {th }}$ iteration, we have identified $n$ critical sites. If the $y$ coordinates of these critical sites are independently distributed, the mean distance between critical sites along $y$ is $L / n$. In fact, a closer inspection of this question shows that the critical sites "repel" each other and thus they tend to be evenly distributed along the $y$ axis. This reinforces the estimate of the mean separation as being $L / n$. The total mass $M(n)$ can thus be expressed as the number of intervals between critical sites, $n$, times the mass of the blobs $(L / n)^{D}$ at the scale of the mean distance. Thus we obtain

$$
M(n) \propto L^{D} n^{1-D}
$$

Using the expression of the fractal dimension, equation (6), the exponent of $n$ amounts to $-\left(\nu_{\perp}-2 \beta\right) / \nu_{\|} \approx-0.31$, close to the measured value -0.28 of Figure 4 . Using the numerical estimate of $D \approx 1.27$ from Figure 3 , then the agreement is excellent.

The insert of Figure 4 shows the evolution of the norse $\eta_{2^{*}(n)}^{\prime}=t(n)$ as a function of $n$. As mentioned earlier, $t(1)$ is related to the directed percolation threshold $t(1)=p_{\mathrm{c}}$. As $n$ 
increases, the noise on the critical site increases to reach 0 at the final stage close to linearly in $n$. The analysis of the data shows that the rate of variation of $t$ with $n$ is proportional to the exponential of the total mass : $\mathrm{d} t(n) / \mathrm{d} n \propto \exp (k M(n))$.

What about the small temperature for Hamiltonian (3)? Being an intensive variable, we expect at any temperatures the entropy will dominate over energy and the ground state behavior cannot persist at any finite $T$, as it is usual also for the extremal dynamic models [10-16]. We can nevertheless discuss the qualitative behavior of the small $T$ behavior of our model (3). For $T \ll 1$, equations (2) and (3) correspond to directed percolation above threshold, $\mathcal{H}(\mathcal{P})>p_{c}$. It has been shown $[19,20]$ that the super-percolation directed paths have the transverse deviation exponent equal to that of directed polymers, i.e. $2 / 3$. So this result indicates that at small enough temperature the scaling exponent jumps from its zero temperature value $\left(\nu_{\perp} / \nu_{\|} \approx 0.63\right)$ to $2 / 3$. This jump in exponent corresponds only to the limit of an infinite system size. For a low temperature, there exist a finite correlation length of the associated directed percolation problem $\left(\xi_{\|} \propto T^{-\nu_{\|}}\right)$. Below $\xi_{\|}$the transverse deviation scales as for $T=0$, whereas at larger length scales the usual directed polymer exponent $(2 / 3)$ is recovered.

Note that for an infinitesimal value of $\alpha$ the ground state is not degenerate and corresponds exactly to the directed line in Figure 1. The progressive optimization introduced above has bypassed the difficulty of dealing with extremely large numbers and permits us to find the ground state for $\alpha=0^{+}$. In this limit of a small but non zero $\alpha$ it is worth considering the small temperature case. In order to do this, one has to go to the definition (4) and then use the fact that $\alpha \ll 1$. Interestingly, a small temperature corresponds to stopping the procedure of degeneracy reduction introduced above at an intermediate stage. Considering paths whose energy difference with that of the ground state is given by $T$, gives rise to the backbone structure optimized down to a threshold $t$ such that $t \approx T^{\alpha}$. It is obvious from this last estimate that taking the limit $T \rightarrow 0$ and $\alpha \rightarrow 0$ give different answers. A critical temperature $T_{\mathrm{c}}$ arises which separates the directed percolation regime to the two dimensional directed polymer $(\alpha=1)$ regime. This $T_{\mathrm{c}}$ scales as $p_{\mathrm{c}}^{1 / \alpha}$ It would be interesting to directly investigate numerically this finite temperature behavior.

\section{Acknowledgments}

S. Roux thanks the Institute for Theoretical Physics of the University of Fribourg for its hospitality during a visit where this work was initiated. We acknowledge inspiring discussions with Hans Herrmann and Amos Maritan. LPMMH is "Unité de Recherche Assoclée" n 857 of the CNRS.

\section{References}

[1] Kardar M. and Zhang Y.-C., Phys. Rev. Lett. 58 (1987) 2087, and see Halpin-Healy T. and Zhang Y.-C., Phys. Rep. 254 (1995) 215 for complete references.

[2] For a review see Kinzel W., in "Percolation structures and process, G. Deutsher, R. Zallen and J. Adler Eds. (Hilger, Bristol, 1983) p. 425.

[3] Sneppen K., Phys. Rev. Lett. 69 (1992) 3539.

[4] Buldyrev S.V., Barabási A.L., Caserta F., Havlın S., Stanley H.E. and Vicsek T., Phys. Rev. A 45 (1992) 8313, and Proceedings of Granada Conf. (Oct, 1991).

[5] Zaitsev S.I., Physica A 189 (1992) 411. 
[6] Kardar M., Parisı G. and Zhang Y.-C., Phys. Rev. Lett. 56 (1986) 889.

[7] Parisi G., Europhys. Lett. 17 (1991) 673.

[8] Roux S. and Hansen A., J. Phys. I France 4 (1994) 515.

[9] Galluccio S. and Zhang Y.-C., Phys. Rev. E 51 (1995) 1686.

[10] Olami Z., Procaccia I. and Zeitak R., Phys. Rev. E 49 (1993) 1232.

[11] Leschhorn H. and Tang L.-H., Phys. Rev. E 49 (1994) 1238.

[12] Bak P. and Sneppen K., Phys. Rev. Lett. 71 (1993) 4083.

[13] Paczuski M., Maslov S. and Bak P., Europhys. Lett. 27 (1994) 97.

[14] Ambegaokar V., Halperin B.I. and Langer J.S., Phys. Rev. B 4 (1971) 2612.

[15] Čieplack M., Maritan A. and Banavar J.R., Phys. Rev. Lett. 72 (1994) 2320.

[16] Roux S., Hansen A. and Guyon E., J. Phys. France 48 (1987) 2125.

[17] Essam J.W., de'Bell K., Adler J. and Bhatti F.M., Phys. Rev. B 33 (1986) 1982.

[18] Arora B.M., Barma M., Dhar D. and Phani M.K., J. Phys. C 16 (1983) 2913.

[19] Balents L. and Kardar M., J. Stat. Phys. 67 (1992) 1.

[20] Lebeder N. and Zhang Y.-C., J. Phys. A 28 (1995) L1. 